\title{
Análisis del perfil emprendedor para la formación de las nuevas generaciones de jóvenes chilenos
}

\author{
Andrés A. Valenzuela-Keller, Francisco A. Gálvez-Gamboa, David R. Contreras, y Felipe P. Parraguez \\ Facultad de Ciencias Sociales y Económicas, Universidad Católica del Maule, Av. San Miguel 3605, Talca, Chile. \\ (correo-e: andresvalenzuelak@gmail.com, franziscogalvez@gmail.com,fpatricio888@gmail.com, \\ davidre.contreras@gmail.com)
}

Recibido Sep. 22, 2020; Aceptado Nov. 18, 2020; Versión final Dic. 4, 2020, Publicado Feb. 2021

\section{Resumen}

El objetivo de este estudio es analizar los perfiles emprendedores de jóvenes chilenos mediante el enfoque de la Teoría de la Motivación utilizando la encuesta "características emprendedoras personales" (CEP). La muestra esta compuesta por 380 jóvenes entre 18 y 25 años de Talca, Chile. Para procesar los datos, se utilizó el análisis clúster mediante el algoritmo de k-medias. Los resultados evidencian la existencia de dos perfiles emprendedores principalmente diferenciados por la edad y la exposición a la actividad empresarial mediante emprendimientos familiares, sugiriendo efectos de la formación emprendedora. Se evidencia que las habilidades de planificación y exigencia de calidad son las más relevantes para explicar el perfil de ambos subgrupos y se sugiere el desarrollo de habilidades transversales como la detección de oportunidades y proactividad. Se concluye que la exposición de los jóvenes a actividades de emprendimiento es favorable y potencia su motivación emprendedora y que la edad es un factor relevante en la formación de los nuevos emprendedores.

Palabras clave: emprendimiento; motivación emprendedora; intención; competencia; educación

\section{Analysis of entrepreneurial profile to train new generations of young Chileans}

\begin{abstract}
The primary objective of this research study is to assess entrepreneurial profiles of young Chileans by using the Motivation Theory approach and the personal entrepreneurship characteristics (PEC) survey. The sample consists of 380 young Chileans (18 to 25 years old) from Talca, Chile. Cluster analysis using the k-means algorithm was used to process and analyze data. The results show the existence of two entrepreneurial profiles that differ mainly by age and by previous exposure to business activity through family ventures. This suggests that there is an impact of previous entrepreneurial training in the sample surveyed. In addition, the results show that planning and quality assurances are the most relevant skills to explain the profiles of both subgroups. This suggests the need to develop transversal skills such as opportunity detection and proactivity. It is concluded that exposure to entrepreneurial activities favors entrepreneurial motivation in young Chileans and that age is a relevant factor for training new entrepreneurs.
\end{abstract}

Keywords: entrepreneurship; entrepreneurial motivation; intention; competence; education 


\section{INTRODUCCIÓN}

En los últimos años, el estudio del emprendimiento y particularmente de las actitudes o intención emprendedora de los jóvenes ha sido abordada ampliamente. No obstante, gran parte de las revisiones realizadas se han focalizado en la Teoría del Comportamiento Planificado (en adelante, TCP) propuesto por Ajzen (1991) que analiza la intención por emprender desde factores como: 1) la actitud; 2) la norma social subjetiva; y 3) el control percibido mediante autoeficacia. Así, gran parte de estos estudios señalan que la intención emprendedora es un predictor de la acción de emprender (McNally et al., 2016). En ese sentido, es que la motivación y características individuales juegan un rol importante dado que interfieren en la decisión del individuo por emprender (Marulanda et al., 2014; Van Hemmen et al., 2013).

Así, la mayor parte de los estudios se han enfocado en la TCP para analizar la intención por emprender, ver por ejemplo Farhat y Guevara (2020); Fragoso et al. (2020) Fietze y Boyd (2017); o Henley et al. (2017). Sin embargo, estos estudios no han profundizado en factores como la motivación, más allá de la percepción de los jóvenes como futuros emprendedores ni mucho menos se han aventurado a analizar si existen diferentes perfiles entre los grupos de jóvenes. Esto último es relevante considerando que autores como Muragesam y Jayavelu (2017) sugieren la importancia de explorar factores como la personalidad o autoeficacia desde enfoques como el motivacional.

En ese contexto, la motivación y su relación con la actividad emprendedora ha sido explorada desde varios enfoques como el plano económico, la búsqueda de oportunidades e inclusive la realización personal (Ferreira et al., 2017). En general, la teoría más utilizada en el enfoque de la motivación es la propuesta por McClelland (1965). Así, autores como Barba-Sánchez y Atienza-Sahuquillo (2012) han explorado los factores asociados a la motivación y como los perfiles motivacionales impactan sobre la decisión de emprender por sobre los relacionados con ganar dinero o ser su propio jefe. Es más, el mismo Ferreira et al. (2017) exploró las motivaciones en estudiantes universitarios, demostrando que aquellos individuos más motivados, son lo que presentaban una mejor intención por la creación de negocios.

En ese sentido, tal como señalan Sivarajah y Achchuthan (2013) la necesidad de logro se presenta como el factor motivacional más fuerte al momento de analizar la intención por emprender. Justamente, este aspecto está muy vinculado al enfoque propuesto por McClelland (1965) y la concepción de que la necesidad de logro en los jóvenes se relaciona con dimensiones asociadas a: metas, motivación, acción y contexto. Consecuentemente, durante los últimos años se han realizado algunos estudios que señalan una relación positiva entre el potencial emprendedor y la motivación, principalmente asociada a la necesidad de logro o autodeterminación de los jóvenes (ver por ejemplo, Al-Jubari, 2019; Barton et al., 2018). No obstante, la discusión de como la motivación individual afecta a la intención emprendedora sigue abierta, sobre todo si consideramos a autores como Raman et al. (2013) que señalan que no existen asociaciones significativas entre motivación e intención por emprender.

Conjuntamente, los estudios del potencial emprendedor han incluido variables sociodemográficas en la explicación de la intención por generar negocios. Por ejemplo, Bakar et al (2017) logró identificar que afectan el potencial emprendedor los recursos financieros e ingreso, las competencias emprendedoras, el género, la educación y el conocimiento de otros empresarios. Adicionalmente, estudios como Bretones y Radrigán (2018) y Herman (2019) señalan la importancia de la formación de los emprendedores en la intención de emprender, notando que los individuos son más propensos a generar emprendimientos cuando son expuestos a estos. De acuerdo a Barba-Sánchez y Atienza-Sahuquillo (2018) o Apolo et al. (2016) la exposición a formación o experiencias emprendedoras genera capacidad de innovación y desarrollo individual, lo que desencadena en mayores probabilidades de emprender, por lo que la detección de competencias emprendedoras es clave en el desarrollo del potencial emprendedor.

Por otro lado, autores como Anggadwita et al. (2017) han incorporado al género como una variable determinante de la intención por emprender, no obstante, son mesurados en reportar que muchas veces la significancia de esta variable obedece a patrones culturales y contextuales. Asimismo, se ha reportado que la capacidad percibida por medio de cómo el individuo se visualiza en el contexto de su ejercicio profesional, también afecta la intención por emprender (Ahmed et al., 2017). Es más, autores como McCann (2017) han demostrado que buena parte de la intención por la generación de negocios depende de las expectativas futuras como emprendedor.

En Chile, autores como Vélez et al. (2020) han analizado desde el enfoque de TCP como la educación emprendedora potencia la intención por emprender. Por otro lado, autores como Soria-Barreto et al. (2016) identificaron mediante la misma teoría que la capacidad percibida y el ingreso familiar afectan significativamente la intención por emprender. El presente estudio aporta a la discusión desde el enfoque de la Teoría de la Motivación propuesta por McClelland (1965) la que ha sido poco abordada para identificar los 
promotores del emprendimiento en los jóvenes y sugiriendo que la motivación de logro interfiere en la decisión de emprender. Además, desde este enfoque se pueden identificar diferentes estrategias para desarrollar las habilidades que potencian esta motivación y repercuten en la intención emprendedora.

Para el desarrollo del estudio, se utilizó la Encuesta Características Emprendedoras Personales (CEP) en una muestra de 400 jóvenes de entre 18 y 25 años de la comuna de Talca, Chile, con el objetivo de identificar la existencia de diferentes perfiles emprendedores. La metodología empleada fue el análisis de clúster o conglomerados a través del algoritmo de k-medias, logrando identificar dos subgrupos o perfiles. Los resultados muestran que las exposiciones de los jóvenes a familiares emprendedores modifican favorablemente su motivación emprendedora y que existen diferencias del desarrollo de está motivación principalmente asociadas a la edad.

Como implicancias, se sugiere desarrollar estrategias particulares entre los diferentes grupos que favorezcan la exposición de los jóvenes a situaciones reales de emprendimientos, pues conocer esta modifica favorablemente la intención y motivación por emprender. Es por ello que se sugiere que en los diferentes niveles educativos puedan existir instancias de vinculación con el mundo empresarial y emprendedores, además del desarrollo de actividades que permitan potenciar las competencias emprendedoras de los jóvenes. Las siguientes secciones desarrollan la metodología empleada, los resultados, la discusión y finalmente las conclusiones vertidas de la investigación realizada.

\section{METODOLOGÍA}

El presente estudio es del tipo descriptivo de corte transversal a través de la aplicación de una encuesta. Las siguientes secciones muestran el detalle de la muestra utilizada, el instrumento y metodología empleada en el procesamiento de los datos.

\section{Participantes y muestra}

En el estudio se utilizó una muestra de jóvenes entre 18 y 25 años de la comuna de Talca, Chile. De acuerdo a cifras de la Encuesta de Caracterización Socioeconómica Nacional (CASEN) del 2017, la población entre los 18 y 25 años en la comuna de Talca asciende a 34.336, por lo que la muestra representativa a un 95\% de confianza y $5 \%$ de error ascendió a 380 individuos. En total, se muestrearon 400 casos mediante la aplicación de cuestionarios con modalidad de autoreporte lo que permite obtener una muestra representativa de la población seleccionada. De la muestra, 164 jóvenes son hombres y 236 mujeres, es decir un $41 \%$ y $59 \%$ respectivamente. Con respecto a la edad, el 58,3\% (233) tienen 18 años; el 26,3\% (105) tienen entre 19 y 21 años; y el 15,4\% (62) tienen entre 22 y 25 años. De estos, un 21,3\% (85) declaró tener ingresos familiares de entre $\$ 350.001$ y $\$ 500.000$; un $19 \%$ (76) menor a $\$ 350.000$; un $18,8 \%$ (75) entre $\$ 1.000 .001$ y $\$ 1.500 .000$; un $17,3 \%$ (69) entre $\$ 500.001$ y $\$ 750.000$; un $14,8 \%$ (59) entre $\$ 750.001$ y $\$ 1.000 .000$; y un $9 \%$ (36) más de $\$ 1.500 .000$.

\section{Instrumento}

Se utilizó la Encuesta Características Emprendedoras Personales (CEP) desarrollada por McClelland (1965) quien desde una perspectiva psicológica y enfoque de competencias analizó el comportamiento empresarial mediante la teoría de las motivaciones. Los factores identificados por McClelland (1965) son: 1) Oportunidades e iniciativa; 2) Persistencia; 3) Cumplimiento; 4) Exigencia de eficiencia y calidad; 5) Correr riesgos calculados; 6) Fijar metas; 7) Búsqueda de información; 8) Planificación sistemática y seguimiento; 9) Persuasión y redes de apoyo; y 10) Autoconfianza e independencia. El cuestionario está conformado por 55 preguntas con una escala de respuestas ordinal de grado de acuerdo del tipo Likert en el rango 1 a 5 . En cuanto antecedentes de validez del instrumento se obtuvo un índice de alpha de Cronbach de 0,920 y dos mitades de Guttman de 0,950 lo que indica alta confiabilidad.

\section{Análisis de clúster}

Se utilizó un método de agrupación considerando su utilidad en el análisis de perfiles (ver por ejemplo, DeLa-Hoz et al, 2018). La estimación utilizó el método de agrupación basado en k-medias (MacQueen, 1967) que permite clasificar los objetos dentro de varios grupos o clúster de tal manera que estos sean similares y representados por un centroide que corresponde a la media de los puntos asignados a su grupo. En esta aplicación, se utilizó un tipo de clúster no jerárquico tomando como referencia la no existencia de una estructura vertical entre los grupos y que el centroide está basado en un prototipo de perfil que define al grupo. Además, se evitó la elección arbitraria de k mediante el procedimiento desarrollado por Charrad et al. (2014) que permite identificar dentro de diferentes índices el valor de $k$ que resulta ser óptimo. De esta manera, se obtuvo que el óptimo resulta de $\mathrm{k}=2$ agrupaciones (como referencia en el análisis 16 índices indicaron 2 
clústeres; 6 indican 3 clústeres; 1 indican 5,7 y 8 clústeres; y 3 indican 10 clústeres). El algoritmo utilizado fue el propuesto por Hartigan y Wong (1979) que define la variación total dentro de un clúster como la suma de las distancias al cuadrado sobre las distancias euclidianas entre elementos y el centroide, tal que:

$$
W\left(C_{k}\right)=\sum_{x_{i} \in C_{k}}\left(x_{i}-\mu_{k}\right)^{2}
$$

Donde, $x_{i}$ diseña un punto de datos pertenecientes al clúster $C_{k}$ y $\mu_{k}$ es el valor medio de los puntos asignados en el clúster $C_{k}$ De esta manera, cada observación de $x_{i}$ se asigna a un grupo dado de tal manera que la distancia de la suma de sus cuadrados de cada observación respecto del centro de cada grupo asignado $\mu_{\mathrm{k}}$ sea minimizado. En la estimación se utilizó el software Rstudio.

\section{RESULTADOS}

A continuación, se muestran los resultados en términos descriptivos, las diferencias entre los dos clústeres identificados y su respectiva caracterización.

\section{Resultados descriptivos}

En la Figura 1 se muestra la distribución del puntaje CEP obtenido por la muestra con media de 17,935 y desviación estándar de 2,097 los que siguen una distribución normal (test Kolmogorov-Smirnov Lillefors para $n>50$ muestra valor de prueba de 0,07 y $p$-valor $>0,05$ ). En la Tabla 1 se muestra las diferencias de medias medida por prueba t y Anova (significancia: ${ }^{*} p<0,1{ }^{* *} p<0,05^{* * *} p<0,01$ ) entre el promedio de la escala CEP y las diferentes variables demográficas incluidas. Se identifican diferencias significativas por género a favor de las mujeres. En la Tabla 1 se puede apreciar que la motivación "Independencia (se propio jefe)" muestra diferencias significativas por sobre otras motivaciones por emprender. También, se logran apreciar diferencias significativas entre quienes tienen familiares emprendedores y quiénes no. Por otro lado, se observan leves diferencias significativas por ingreso mostrando que quienes tienen mayor ingreso familiar tienen una apreciación mayor por emprender. Finalmente, en cuanto a los rangos de edad se observan diferencias entre los que pertenecen a los tramos 18 años (primer año universitario) y 22 a 25 años, a favor de estos últimos.

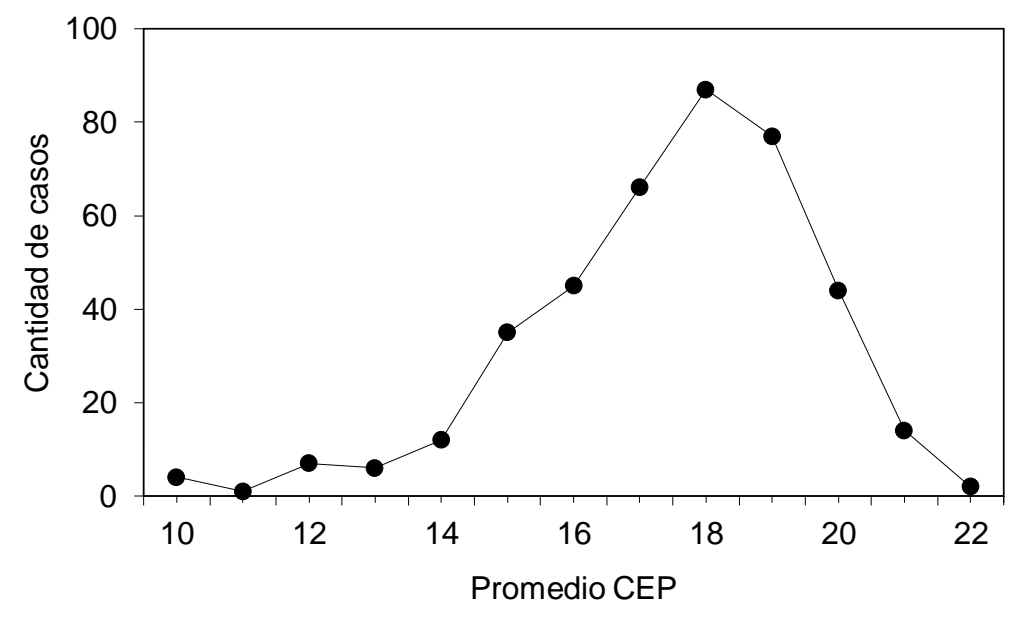

Figura 1: Distribución Puntaje CEP (promedio) y número de casos.

Tabla 1: Diferencia CEP (promedio) y Variables

\begin{tabular}{|l|l|l|c|c|c|}
\hline \multicolumn{2}{|c|}{} & \multicolumn{2}{c|}{ Media } & \multicolumn{2}{c|}{ Diferencia } \\
\hline \multirow{3}{*}{ Género: } & 1 & Hombre & 17,779 & $2>1$ & $0,265^{* * *}$ \\
\cline { 2 - 6 } & 2 & Mujer & 18,044 & \multicolumn{2}{|c|}{} \\
\hline \multirow{3}{*}{ Motivación por emprender: } & 1 & Ganar dinero & 17,63 & $5>1$ & $0,705^{\star}$ \\
\cline { 2 - 6 } & 2 & Demostrar que eres capaz & 17,557 & $5>2$ & $0,778^{\star}$ \\
\cline { 2 - 6 } & 3 & Salir del desempleo & 17,375 & & \\
\hline & 4 & Presión por la familia & 17,333 & & \\
\hline
\end{tabular}


Tabla 1: continuación.

\begin{tabular}{|c|c|c|c|c|c|}
\hline & & & Media & \multicolumn{2}{|c|}{ Diferencia } \\
\hline \multirow{2}{*}{ Motivación por emprender: } & 5 & Independencia (se propio jefe) & 18,336 & & \\
\hline & 6 & No está en mis objetivos emprender & 17,905 & & \\
\hline \multirow{2}{*}{ Familiar emprendedor: } & 1 & Si & 18,242 & $1>2$ & $0,585^{* * *}$ \\
\hline & 2 & No & 17,657 & & \\
\hline \multirow{6}{*}{ Ingreso familiar: } & 1 & Hasta $\$ 350.000$ & 17,834 & $6>3$ & $0.894^{*}$ \\
\hline & 2 & Entre $\$ 350.001$ y $\$ 500.000$ & 17,759 & & \\
\hline & 3 & Entre $\$ 500.001$ y $\$ 750.000$ & 17,613 & & \\
\hline & 4 & Entre $\$ 750.001$ y $\$ 1.000 .000$ & 17,807 & & \\
\hline & 5 & Entre $\$ 1.000 .001$ y $\$ 1.500 .000$ & 18,2 & & \\
\hline & 6 & Más de $\$ 1.500 .000$ & 18,508 & & \\
\hline \multirow{3}{*}{ Rango de edad: } & 1 & 18 años & 17,729 & $3>1$ & $0,669^{*}$ \\
\hline & 2 & 19 a 21 años & 18,119 & & \\
\hline & 3 & 22 a 25 años & 18,398 & & \\
\hline
\end{tabular}

\section{Diferencias CEP entre clúster}

En la Tabla 2 se muestra las diferencias de medias con prueba $t$ (significancia: ${ }^{*} p<0,1{ }^{* *} p<0,0{ }^{* * *} p<0,01$ ) entre dimensiones de la encuesta CEP y los dos clústeres identificados. Cabe mencionar que se probó normalidad de las dimensiones CEP utilizando la prueba Kolmogorov-Smirnov Lillefors (detalle en Tabla 2). En términos generales, se puede observar diferencias significativas en todos los ítems específicos de la medición realizada, por lo que los individuos del clúster 2 presentan un perfil más orientado a características emprendedoras. Las dimensiones que muestran mayores diferencias en cuantía son "Fijar metas" (diferencia de 4,442 puntos) y "Tener autoeficacia" (diferencia de 4,220 puntos). Por otro lado, la dimensión que muestra menor diferencia entre los clústeres es "Correr riesgos" (diferencia de 2,832 puntos).

Tabla 2: Diferencia CEP (por dimensión) y Clúster

\begin{tabular}{|l|c|c|c|c|c|c|c|c|}
\hline & \multirow{2}{*}{$\begin{array}{c}\text { K-S } \\
\text { Lillefors }\end{array}$} & \multicolumn{3}{|c|}{ Clúster 1 } & \multicolumn{3}{c|}{ Clúster 2 } \\
\cline { 3 - 7 } & & Media & $\begin{array}{c}\text { Desv. } \\
\text { Estándar }\end{array}$ & Mediana & Media & $\begin{array}{c}\text { Desv. } \\
\text { Estándar }\end{array}$ & Mediana & Diferencia \\
\hline $\begin{array}{l}\text { CEP 1: Búsqueda de } \\
\text { oportunidad e iniciativa }\end{array}$ & 0,101 & 14,200 & 2,601 & 14,000 & 17,585 & 2,157 & 18,000 & $3,385^{* * *}$ \\
\hline CEP 2: Ser persistente & 0,073 & 16,069 & 2,671 & 16,000 & 20,152 & 2,301 & 20,000 & $4,083^{\star * *}$ \\
\hline $\begin{array}{l}\text { CEP 3: Ser fiel al } \\
\text { cumplimiento del contrato de } \\
\text { trabajo }\end{array}$ & 0,095 & 15,923 & 2,946 & 16,000 & 18,959 & 2,333 & 19,000 & $3,036^{\star * *}$ \\
\hline $\begin{array}{l}\text { CEP 4: Exigir eficiencia y } \\
\text { calidad }\end{array}$ & 0,137 & 15,508 & 2,829 & 16,000 & 18,741 & 2,210 & 19,000 & $3,233^{* * *}$ \\
\hline CEP 5: Correr riesgos & 0,107 & 14,546 & 2,910 & 15,000 & 17,378 & 2,238 & 17,000 & $2,832^{\star * *}$ \\
\hline CEP 6: Fijar metas & 0,115 & 16,769 & 3,109 & 17,000 & 21,211 & 2,657 & 21,500 & $4,442^{\star * *}$ \\
\hline $\begin{array}{l}\text { CEP 7: Conseguir } \\
\text { información }\end{array}$ & 0,103 & 16,877 & 2,745 & 17,000 & 20,185 & 2,348 & 20,000 & $3,308^{\star * *}$ \\
\hline $\begin{array}{l}\text { CEP 8: Planificar y controlar } \\
\text { sistemáticamente }\end{array}$ & 0,096 & 15,246 & 2,576 & 15,000 & 18,344 & 2,474 & 19,000 & $3,098^{* * *}$ \\
\hline $\begin{array}{l}\text { CEP 9: Ser persuasivo y } \\
\text { crear redes de apoyo }\end{array}$ & 0,089 & 14,346 & 2,681 & 15,000 & 18,274 & 2,422 & 18,000 & $3,928^{* * *}$ \\
\hline $\begin{array}{l}\text { CEP 10: Tener } \\
\text { autoconfianza }\end{array}$ & 0,109 & 15,862 & 2,869 & 16,000 & 20,081 & 2,428 & 20,000 & $4,220^{\star * *}$ \\
\hline
\end{tabular}




\section{Caracterización entre clústeres}

En la Tabla 3 se muestra las diferencias de proporciones en la composición de cada clúster medida por prueba Chi cuadrada (significancia: ${ }^{*} p<0,1{ }^{* *} p<0,05{ }^{* * *} p<0,01$ ). Los resultados muestran las divergencias de caracterización entre los clústeres pudiendo observar diferencias significativas entre la presencia de familiares emprendedores y el tramo de edad de los individuos que lo componen. Estos resultados sugieren que la exposición de los jóvenes a la actividad emprendedora es favorable para el potencial emprendedor. Por otro lado, se sugiere diferencias entre grupos etarios, notando que el primer clúster está compuesto principalmente por individuos más jóvenes y el segundo es más homogéneo.

Tabla 3: Caracterización entre Clústeres

\begin{tabular}{|c|c|c|c|c|}
\hline & & Clúster 1 & Clúster 2 & $x^{2}$ \\
\hline \multirow{2}{*}{ Género: } & Hombre & $42,3 \%$ & $40,4 \%$ & \multirow[t]{2}{*}{0,068} \\
\hline & Mujer & $57,7 \%$ & $59,6 \%$ & \\
\hline \multirow{6}{*}{ Motivación por emprender: } & Ganar dinero & $30,0 \%$ & $25,9 \%$ & \multirow[t]{6}{*}{5,453} \\
\hline & Demostrar que eres capaz & $18,5 \%$ & $17,0 \%$ & \\
\hline & Salir del desempleo & $1,5 \%$ & $0,7 \%$ & \\
\hline & Presión por la familia & $3,8 \%$ & $1,5 \%$ & \\
\hline & Independencia (se propio jefe) & $36,2 \%$ & $45,9 \%$ & \\
\hline & No está en mis objetivos emprender & $10,0 \%$ & $8,9 \%$ & \\
\hline \multirow{2}{*}{ Familiar emprendedor: } & $\mathrm{Si}$ & $36,9 \%$ & $52,2 \%$ & \multirow[t]{2}{*}{$8,023^{\star \star *}$} \\
\hline & No & $63,1 \%$ & $47,4 \%$ & \\
\hline \multirow{6}{*}{ Ingreso familiar: } & Hasta $\$ 350.000$ & $20,8 \%$ & $18,1 \%$ & \multirow[t]{6}{*}{6,338} \\
\hline & Entre $\$ 350.001$ y $\$ 500.000$ & $23,8 \%$ & $20,0 \%$ & \\
\hline & Entre $\$ 500.001$ y $\$ 750.000$ & $18,5 \%$ & $16,7 \%$ & \\
\hline & Entre $\$ 750.001$ y $\$ 1.000 .000$ & $16,9 \%$ & $13,7 \%$ & \\
\hline & Entre $\$ 1.000 .001$ y $\$ 1.500 .000$ & $7,7 \%$ & $9,6 \%$ & \\
\hline & Más de $\$ 1.500 .000$ & $12,3 \%$ & $21,9 \%$ & \\
\hline \multirow{3}{*}{ Rango de edad: } & 18 años & $66,9 \%$ & $54,1 \%$ & \multirow[t]{3}{*}{$5,957^{* *}$} \\
\hline & 19 a 21 años & $20,8 \%$ & $28,9 \%$ & \\
\hline & 22 a 25 años & $12,3 \%$ & $17,0 \%$ & \\
\hline
\end{tabular}

\section{DISCUSIÓN}

En primer lugar, se analizan las diferencias de la escala CEP total promedio notando que existen diferencias de género, resultado que se contrapone a lo que sugieren Soria-Barreto et al. (2016) asociado a la intención por emprender bajo el enfoque de la TCP. Este resultado también es concordante con la evidencia planteada por Anggadwita et al. (2017) o Bakar et al. (2017) respecto a las diferencias de género. Por otro lado, se identificaron diferencias entre ingresos familiares, tal como sugieren Bakar et al (2017), situación importante al notar las recurrentes brechas por ingreso a nivel nacional.

Además, se observan diferencias entre la motivación por emprender, tal como sugieren gran parte de los estudios basados en la TCP mostrando convergencia con éstos (ver por ejemplo, Farhat y Guevara, 2020; Fragoso et al., 2020). También, se identifican diferencias por la existencia de familiares emprendedores lo que sugiere que la exposición a estos modifica la motivación por emprender. Este resultado es concordante con la evidencia planteada por Herman (2019) y Bretones y Radrigán (2018) sobre el impacto de la educación y tradición familiar emprendedora.

En segundo lugar, se discuten los resultados en términos de las diferencias entre clústeres. En ese contexto, se logra identificar que la subdimensión que produce mayor diferencia es la de "Fijar metas" (4,442 puntos con p-valor $<0,05)$, seguido de "Tener autoconfianza" $(4,220$ puntos con $p$-valor $<0,05)$ y "Ser persistente" $(4,083$ puntos con $p$-valor $<0,05)$. Estas refieren de acuerdo a McClelland (1965) a las competencias alusivas 
a subdivisión de tareas, planes y fijación de metas; autorrealización y confianza en sí mismo; y hacer las cosas de forma eficiente buscando excelencia y cumplimiento de lo establecido. Por consiguiente, estas competencias son características de cada clúster que permitirían reforzar estrategias de trabajo particulares con cada grupo.

En tercer lugar, respecto de las características del clúster se denotan un efecto de la experiencia por la edad y conocimiento de emprendedores a través de un familiar (detalle en Tabla 3). En consecuencia, las estrategias en los subgrupos de jóvenes deberían ir enfocadas en los grupos por edad y se releva la necesidad de exposición a situaciones de emprendimiento. En este sentido, estrategias a evaluar en cada grupo surgen de las dimensiones evaluadas en un menor nivel de desarrollo. Así, en el primer clúster se presentan valores menores en "Búsqueda de oportunidad e iniciativa" (media de 14,200) y "Ser persuasivo y crear redes de apoyo" (media de 14,346) por lo que las estrategias en los subgrupos de jóvenes deberían ir enfocadas en desarrollar competencias que permitan aprovechar oportunidades, persuadir a otros y plantear sus ideas. En el caso del segundo clúster, la dimensión evaluada de manera más baja es "Correr riesgos" (media de 17,378) y "Búsqueda de oportunidad e iniciativa" (media de 17,585). Lo anterior, sugiriere estrategias referidas a trabajar competencias como la evaluación de alternativas y el control de resultados.

\section{CONCLUSIONES}

De acuerdo a los resultados del estudio y la discusión realizada, se pueden resumir los siguientes hallazgos: 1) la exposición de los jóvenes a actividades de emprendimiento es favorable y potencia su motivación emprendedora; y 2) la edad es un factor relevante en la formación de los nuevos emprendedores, por lo cual deben generarse estrategias particulares en los diferentes niveles educativos que permitan a estos desarrollar habilidades que les consientan emprender en el futuro.

Como sugerencias de estrategias a desarrollar con los jóvenes en distintos niveles educativos, en primer lugar, se sugiere desarrollar competencias asociadas a la persuasión y planteamiento de ideas en estudiantes de educación media, tomando como referencia los resultados de los estudiantes de primer año (principalmente del clúster 1). Por otro lado, con los grupos de jóvenes mayores o de nivel universitario (clúster 2), se propone estrategias que permitan potenciar habilidades vinculadas a la gestión de riesgos. Finalmente, se identifica como transversal el trabajar las competencias referidas al desarrollo de habilidades en cuanto a detección de oportunidades y proactividad y generar estrategias de vinculación con el mundo emprendedor, tomando como referencia el impacto de la variable familiar emprendedor sobre la intención por emprender.

\section{REFERENCIAS}

Ahmed, T., Chandran, V. G. R., y Klobas, J., Specialized entrepreneurship education: does it really matter? Fresh evidence from Pakistan, http://dx.doi.org/10.1108/lJEBR-01-2016-0005, International Journal of Entrepreneurial Behavior $y$ Research, 23(1), 4-19 (2017).

Ajzen, I., The theory of planned behavior, https://doi.org/10.1016/0749-5978(91)90020-T, Organizational Behavior and Human Decision Processes, 50(2), 179-211 (1991).

Al-Jubari, I., College students' entrepreneurial intention: testing an integrated model of SDT and TPB, https://doi.org/10.1177\%2F2158244019853467, Sage Open, 9(2) (2019).

Anggadwita, G., Luturlean, B. S., Ramadani, V., y Ratten, V., Socio-cultural environments and emerging economy entrepreneurship: women entrepreneurs in Indonesia, https://doi.org/10.1108/JEEE-03-2016-0011, Journal of Entrepreneurship in Emerging Economies, 9(1), 85-96 (2017).

Apolo, M. B., Molina, A. P., Orellana, B. S., y Brito-Gaona, L., Influencia del nivel de instrucción en la capacidad emprendedora, https://doi.org/10.19044/esj.2016.v12n25p131, European Scientific Journal, 12(25) (2016).

Bakar, A. R. A., Ahmad, S. Z., Wright, N. S., y Skoko, H., The propensity to business startup: evidence from Global Entrepreneurship Monitor (GEM) data in Saudi Arabia, https://doi.org/10.1108/JEEE-11-2016-0049, Journal of Entrepreneurship in Emerging Economies, 9(3), 263-285 (2017).

Barba-Sánchez, V., y Atienza-Sahuquillo, C., Entrepreneurial behavior: Impact of motivation factors on decision to create a new venture, https://doi.org/10.1016/S1135-2523(12)70003-5, Investigaciones Europeas de Dirección y Economía de la Empresa, 18(2), 132-138 (2012).

Barba-Sánchez, V., y Atienza-Sahuquillo, C., Entrepreneurial intention among engineering students: The role of entrepreneurship education, https://doi.org/10.1016/j.iedeen.2017.04.001, European Research on Management and Business Economics, 24(1), 53-61 (2018).

Barton, M., Schaefer, R., y Canavati, S., To be or not to be a social entrepreneur: motivational drivers amongst American business students, https://doi.org/10.15678/EBER.2018.060101, Entrepreneurial Business and Economics Review, 6(1), 9-35 (2018). 
Bretones, F. D., y Radrigán, M., Actitudes hacia el emprendimiento: el caso de los estudiantes universitarios chilenos y españoles, https://doi.org/10.7203/CIRIEC-E.94.12668, CIRIEC-España, (94), 11-30 (2018).

Charrad M., Ghazzali N., Boiteau V., y Niknafs A., NbClust: an R package for determining the relevant number of clusters in a Data Set, https://doi.org/10.18637/jss.v061.i06, Journal of Statistical Software, 61(6), 1-36 (2014).

De-La-Hoz, E. J., De-La-Hoz, E. J., y Fontalvo, T. J., Metodología de aprendizaje automático para la clasificación y predicción de usuarios en ambientes virtuales de educación, https://dx.doi.org/10.4067/S071807642019000100247, Información tecnológica, 30(1), 247-254 (2019).

Farhat, S. D., y Guevara, R., Incidencia de la actitud conductual en las intenciones emprendedoras en estudiantes universitarios, https://doi.org/10.22201/fca.24488410e.2019.2074, Contaduría y administración, 65(2) (2020).

Ferreira, A. D. S. M., Loiola, E., y Gondim, S. M. G., Motivations, business planning, and risk management: entrepreneurship among university students, https://doi.org/10.1016/j.rai.2017.03.003, RAI Revista de administracao e inovacao, 14(2), 140-150 (2017).

Fietze, S., y Boyd, B., Entrepreneurial intention of Danish students: a correspondence analysis, https://doi.org/10.1108/IJEBR-08-2016-0241, International Journal of Entrepreneurial Behavior \& Research, 23(4), 656672 (2017).

Fragoso, R., Rocha-Junior, W., y Xavier, A., Determinant factors of entrepreneurial intention among university students in Brazil and Portugal, https://doi.org/10.1080/08276331.2018.1551459, Journal of Small Business \& Entrepreneurship, 32(1), 33-57 (2020).

Hartigan, J. A.; y Wong, M. A., Algorithm AS 136: A k-medias clustering clgorithm, https://doi.org/10.2307/2346830, Journal of the Royal Statistical Society, Series C (Applied Statistics), 28(1), 100-108 (1979).

Henley, A., Contreras, F., Espinosa, J. C., y Barbosa, D., Entrepreneurial intentions of Colombian business students, https://doi.org/10.1108/IJEBR-01-2017-0031, International Journal of Entrepreneurial Behavior \& Research, 23(6), 10171032 (2017).

Herman, E., Entrepreneurial intention among engineering students and its main determinants, https://doi.org/10.1016/j.promfg.2019.02.220, Procedia Manufacturing, 32, 318-324 (2019).

MacQueen, J. B., Some methods for classification y analysis of multivariate observations. Proceedings of 5th Berkeley Symposium on Mathematical Statistics and Probability 1. University of California Press. pp. 281-297 (1967).

McCann, B. T., Prior exposure to entrepreneurship and entrepreneurial beliefs, https://doi.org/10.1108/IJEBR-05-20160160, International Journal of Entrepreneurial Behavior y Research, 23(3), 591-612 (2017).

McClelland, D. C., N achievement y entrepreneurship: a longitudinal study, https://doi.org/10.1037/h0021956, Journal of personality and Social Psychology, 1(4), 389 (1965).

McNally, J. J., Martin, B. C., y otros tres autores, Toward rigor and parsimony: a primary validation of Kolvereid's (1996) entrepreneurial attitudes scales, https://doi.org/10.1080/08985626.2016.1154985, Entrepreneurship \& Regional Development, 28(5-6), 358-379 (2016).

Marulanda, F. Á., Montoya, I. A., y Vélez, J. M., Teorías motivacionales en el estudio del emprendimiento, https://doi.org/10.14482/pege.36.5571, Pensamiento \& Gestión, 36, 206-238 (2014).

Murugesan, R., y Jayavelu, R., The influence of big five personality traits and self-efficacy on entrepreneurial intention: The role of gender, https://doi.org/10.1177/2393957516684569, Journal of entrepreneurship and innovation in emerging economies, 3(1), 41-61 (2017).

Raman, K., Anantharaman, R. N., y Ramanathan, S., Environmental, personality and motivational factors: a comparison study between women entrepreneurs and women non entrepreneurs in Malaysia, https://doi.org/10.5539/ijbm.v8n13p15, International journal of business and management, 8(13), 15 (2013).

Sivarajah, K., y Achchuthan, S., Entrepreneurial Intention among Undergraduates: review of literature, European Journal of Business and Management, ISSN: 2222-1905, 5(5), 172-186 (2013).

Soria-Barreto, K., Zuniga-Jara, S., y Ruiz-Campo, S., Educación e intención emprendedora en estudiantes universitarios: un caso de estudio, https://doi.org/10.4067/S0718-50062016000100004, Formación universitaria, 9(1), 25-34 (2016).

Van Hemmen, S., Urbano, D., y Alvarez, C., Charismatic leadership and entrepreneurial activity: An empirical analysis, Innovar, ISSN: 0121-5051, 23(50), 53-65 (2013).

Vélez, C. I., Bustamante, M. A., Loor, B. A., y Afcha, S. M., La educación para el emprendimiento como predictor de una intención emprendedora de estudiantes universitarios, https://doi.org/10.4067/S0718-50062020000200063, Formación universitaria, 13(2), 63-72 (2020). 